\title{
Redes neurais artificiais paraconsistentes aplicadas no monitoramento óptico de produtos
}

\section{Paraconsistent artificial neural networks applied in the optical monitoring of goods}

\author{
João Luís Lopes Freitas Orsi Kuntz ${ }^{(1}$ and Maurício Conceição Mário ${ }^{\oplus}, 1$ \\ ${ }^{1}$ Universidade Santa Cecília \\ *joaoluisfreitas@gmail.com; cmario@unisanta.br
}

Recebido: 09/03/2021. Revisado: 01/11/2021. Aceito: 23/11/2021.

\begin{abstract}
Resumo
Este trabalho apresenta o uso da Lógica Paraconsistente (LP) aliada a técnicas da fotogrametria voltadas ao processamento de imagens para obtenção de dados de interesse em um cenário de controle de qualidade de linha de produção. A fotogrametria tem sido aplicada com sucesso em cenários de monitoramento, enquanto a LP, por sua vez, tem sido aplicada em situações de controle de processo. Uma vez que a LP considera a incerteza ao realizar cálculos, ela se torna útil em cenários onde a medição desempenha um papel fundamental. Aqui são descritas as etapas para a montagem e execução de uma estrutura lógica denominada Unidade de Convolução Paraconsistente (UCP). A UCP é capaz de realizar transformações em imagens e extrair propriedades, utilizadas pelas Células Neurais Artificiais Paraconsistentes (CNAPs) em etapas de classificação dos objetos presentes nas imagens e identificação de desvios em suas dimensões. Os resultados foram encorajadores, apresentando uma diminuição da variância na distribuição de frequência dos níveis de cinza na imagem 13 vezes maior do que a alcançada pelo uso de uma biblioteca de programação padrão, a realização de medidas em objetos com um erro máximo observado de 1,77\% em relação ao valor teórico esperado, uma taxa de precisão de $100 \%$ no processo de classificação de objetos com o uso de algoritmos de baixo uso computacional e a capacidade de identificar desvios nas dimensões do objeto de até $1 \mathrm{~mm}$.
\end{abstract}

Palavras-Chave: Lógica Paraconsistente; Redes Neurais Artificiais Paraconsistentes; Visão Computacional

\begin{abstract}
This work presents the use of the Paraconsistent Logic (PL) combined with techniques of the photogrammetry aimed at processing images to obtain data of interest in a production line quality control scenario. Photogrammetry has been successfully applied in monitoring structures while PL, in turn, has been applied in process control circumstances. Since PL considers uncertainty when performing calculations, it is useful in scenarios where measurement plays a key role. Here, the steps for assembling and executing a logical structure denoted the Paraconsistent Convolution Unit (PCU) are described. PCU performs transformations in images and extracts properties, used by Paraconsistent Artificial Neural Cells for classifying the objects present in the images and identifying deviations in their dimensions. Results were encouraging, presenting a decrease of the variance in the frequency distribution of grey levels in the image 13 times higher than that achieved by the use of a standard programming library, the realization of measurements on objects with an observed maximum error of $1.77 \%$ in relation to the expected theoretical value, a $100 \%$ accuracy rate in the object classification process with the use of low computational-intensive algorithms and an ability to identify deviations in dimensions of the object as low as $1 \mathrm{~mm}$.
\end{abstract}

Keywords: Computer Vision; Paraconsistent Artificial Neural Networks; Paraconsistent Logics 


\section{Introdução}

O surgimento das atividades de monitoramento, controle e garantia da qualidade como atividades apartadas da linha de produção na fabricação de bens diversos foi resultado de um longo processo histórico, paralelo ao desenvolvimento das sociedades. Se a produção "sob medida" foi a regra até fins do século XIX (Paladini, 2011), hoje, em um cenário em que a capacidade de produção em massa possui peso elevado no sucesso das indústrias - salvo nichos específicos - a existência de meios de gerenciamento dos processos torna-se premente. Corolário direto disto foi o desenvolvimento da administração como ciência e o surgimento dos trabalhos sobre a racionalização do trabalho e os estudos sobre tempos e movimentos de Taylor, dos controles de produção de Gantt e da departamentalização de Fayol (Paladini, 2011). Consequência deste movimento, a "inspeção do serviço" desponta como atividade descentralizada.

Apesar de não ser uma ciência recente, o processo de controle de qualidade é cada vez mais valioso dentro de um contexto de mercado global, com grande competitividade, em que fatores como a inovação, a eficácia e eficiência tanto do produto como de seus processos produtivos são relevantes.

Considerando que uma das premissas do Controle Estatístico de Processo (CEP) de Shewhart, surgido entre as décadas de 1920 e 1930, é o de que "a variabilidade deve ser controlada" (Martinelli, 2009), a atividade de monitoramento do processo ganha importância. Neste ínterim, a utilização de técnicas automatizadas, não-destrutivas e de pouco ou nenhum contato com o produto, como é o caso das técnicas ópticas - e entre elas a fotogrametria também ganham em expressividade.

Conclusões tomadas baseadas em técnicas de monitoramento óptico fundamentam-se no reconhecimento de padrões e identificação de desvios, quando ocorrem, e, aqui, a execução de algoritmos computadorizados possui importante aplicação. As Redes Neurais Artificiais (RNAs) são um exemplo das técnicas da informática utilizadas neste processo já há alguns anos, enquanto que as $\mathrm{Re}-$ des Neurais Artificiais Paraconsistentes (RNAPs) são uma classe de RNAs desenvolvidas a partir dos trabalhos de Da Silva Filho (2001) e que se baseiam na utilização da Lógica Paraconsistente (LP), cuja principal característica é a de aceitar a contradição como proposição lógica, considerando variáveis não-lineares, incertas, imprecisas ou mesmo inconsistentes (Da Silva Filho et al., 2008).

Enquanto trabalhos sobre a utilização da LP em análises estatísticas em âmbito industrial e na análise e tomada de decisão na escolha de materiais a serem montados em protótipos de pré-produção - tema abordado, por exemplo, por Gonçalves (2019), no âmbito de uma empresa montadora de veículos - vêm apresentando resultados satisfatórios, a utilização de RNAPs também vem sendo explorada na realização do CEP em processos industriais, tal como descrito por (Rocha, 2019), assim como no de reconhecimento de caracteres em imagens (Souza, 2013), abrindo possibilidades para seu uso na área da visão computacional.

Importante técnica aplicada no processamento de imagens e utilizada no monitoramento óptico em processos industriais, a "fotogrametria" se refere a um conjunto de métodos que se utilizam da luz através de dispositivos, tais como câmeras de vídeo e/ou de fotografia, para a realização de medições e para a estimação de propriedades geométricas e semânticas com base em imagens ou observações por meio de um ou mais dispositivos similares (Stachniss, 2020). Seu termo foi utilizado academicamente pela primeira vez em 1867 por Albrecht Meydenbauer (Albertz, 2001) e tem ganhado especial relevância graças às recentes evoluções em áreas de visão computacional, aprendizagem de máquina e a robótica.

Publicações acadêmicas recentes demonstraram seu uso com sucesso na modelagem topográfica de crateras e rochas lunares na missão chinesa Chang'E-4 com precisão na ordem dos centímetros (Wu et al., 2021), como também utilizaram sistemas robóticos de estereofotogrametria e técnicas de nuvens de pontos para a realização de inspeções em superfícies curvas (Wang et al., 2021). Outras propriedades geométricas, capturadas de superfícies não-lineares, também foram trabalhadas, como no trabalho de Narvydas and Puodziuniene (2020), utilizando imagens binárias (imagens compostas unicamente pelas cores preta e branca).

Na Itália, imagens aéreas foram utilizadas para a realização de inspeções em pontes de concreto, monitoradas para deterioração visual e para acompanhamento de medições periódicas (Zollini et al., 2020). Sistemas aéreos não-tripulados também foram utilizados para a realização de inspeções metrológicas em 3D (Menna et al., 2020).

$\mathrm{Na}$ área da topologia, o processamento de imagens com uso da fotogrametria vem permitido, por exemplo, o controle de variações longitudinais na morfologia de canais no norte da China (Tan et al., 2021), assim como o acompanhamento das instabilidades em declives, através do sensoriamento remoto (Tarolli et al., 2020). Operações de deslocamento lateral (Guidi et al., 2020), inspeções em estruturas de forma não-destrutiva (El Masri and Rakha, 2020) e o monitoramento da estrutura de túneis, com resolução de 0,01 mm (Odumosu et al., 2020) também foram relatados academicamente nos últimos anos.

A fotogrametria foi aplicada com sucesso em sistemas de medição de peças manufaturadas automotivas com formas complexas (Catalucci et al., 2020), assim como foram desenvolvidos estudos sobre seu uso no monitoramento de eficácia e eficiência do controle dimensional industrial (Ma et al., 2021), o que demonstra a viabilidade deste estudo.

O uso de lógicas não-clássicas, por sua vez, aplicadas no monitoramento de qualidade em um CEP foi explorado a partir de modelos baseados na lógica Fuzzy (Boroujeni et al., 2021).

Apesar de a união da LP com a fotogrametria possuir aplicabilidade bastante extensa (pese que ambos os assuntos tenham sido arduamente trabalhados em separado nos últimos anos), a literatura acadêmica é carente de publicações. Identifica-se, aqui, uma oportunidade de desenvolvimento científico importante que permitirá sua continuidade em aplicações reais, com impactos positivos para a sociedade.

O objetivo principal deste trabalho é o de demonstrar a utilização de uma RNAP no processamento de imagens, capaz de extrair atributos e auxiliar na tomada de decisões, com base em técnicas empregadas na fotogrametria, atra- 
vés da descrição do arranjo em diferentes conformações de Células Neurais Artificiais Paraconsistentes (CNAPs), do processo de extração dos atributos de interesse a partir das imagens coletadas, demonstrando o uso da LP em processos de classificação e identificação de desvios.

\section{Referencial Teórico}

\subsection{Lógica Paraconsistente}

Pertencente ao rol das lógicas não clássicas, a LP ganha relevância no século XX a partir dos trabalhos de Newton da Costa, matemático brasileiro que, baseando-se em pesquisas de Lukasiewicz e Vasilév sobre sistemas lógicos que aceitam a contradição como um princípio lógico, elaborou a teoria que a originou, tendo seu nome cunhado apenas alguns anos mais tarde, em 1976, por Francisco Miró Quesada Cantuarias, durante simpósio ocorrido na Universidade Estadual de Campinas.

A LP tem a capacidade de trabalhar com situações em que uma proposição e sua negação possam ser consideradas verdadeiras e que seja possível incluir dois outros estados lógicos à lógica clássica: a inconsistência $(T)$ e a indeterminação $(\perp)$.

Durante década de 1990, a Lógica Paraconsistente Anotada (LPA) - um desdobramento da LP - foi apresentada com a publicação de trabalho de Da Costa em conjunto com Subrahmanian e Vago. Ela considera os quatro estados lógicos da LP dentro do conjunto $\tau=T, V, F, \perp$ e os representa em um reticulado finito em um diagrama de Hasse como cada um de seus vértices. Nesta representação, uma proposição $P$ é acompanhada com a anotação (ou valor correspondente) composta por um grau de evidência favorável $(\mu)$, uma constante anotacional do reticulado, que indica de forma a conotação lógica de determinada proposição.

A Lógica Paraconsistente Anotada com anotação de 2 valores (LPA2v), por sua vez, diferencia-se da LPA principalmente pelo fato de considerar, além do grau de evidência favorável, um grau de evidência desfavorável $(\lambda)$, que rejeita a proposição - este, independente do primeiro. Neste caso, o reticulado finito de Hasse é definido por pares ordenados de acordo com a relação $\tau=(\mu, \lambda) \mid \mu, \lambda \in[0,1] \subset R$, sendo válida a proposição $(\mu, \lambda)=(\lambda, \mu)$, em que $\mu, \lambda \in[0,1]$ em que cada um dos vértices do reticulado de Hasse é representado por um par ordenado no intervalo $[0,1]$, tal como mostrado na Fig. 1. Nele, os vértices representam os valores-limites de cada par ordenado.

A aplicação de transformações lineares em um Quadrado Unitário no Plano Cartesiano (QUPC) mostra válida a transformação $T(X, Y)=(x-y, x+y-1)$ e permite, como consequência, a definição dos termos "Grau de Certeza" $\left(G_{c}\right)$ e "Grau de Contradição" $\left(G_{c t}\right)$, definidos nas Eqs. (1) e (2), pertencentes ao conjunto, $\Re$ e representados no reticulado de Hasse nos eixos horizontal e vertical, respectivamente.

$$
G_{c}=\mu-\lambda
$$

$$
G_{c t}=\mu+\lambda-1
$$

Qualquer valor de $G_{c t}$ diferente de zero implica a existência de um valor máximo para $G_{c}$ diferente do grau máximo possível de certeza, isto é, 1. Este valor, determinado por $\phi$, chamado de "intervalo de certeza", é calculado conforme a Eq. (3) e seus efeitos sobre os valores máximos e mínimos de $G_{c}$ equivalem, respectivamente, $\mathrm{a}+\phi \mathrm{e}-\phi$.

$$
\phi=1-\left|G_{c t}\right|
$$

O resultado desejável de uma análise que utilize a LPA2v deve representar o valor de certeza não contaminado pelos efeitos das incertezas - capaz, portanto, de subtrair o efeito das inconsistências originadas pelas informações conflitantes do grau de certeza das observações.

Quando um par de graus de evidência $\mu$ e $\lambda$ é plotado em um reticulado de Hasse, alguns valores de controle tornam-se importantes, como os casos dos valores superiores e inferiores possíveis para a certeza dada a contradição. Estes são definidos como o Valor Superior de Controle de Certeza, $V_{s c c}$, o Valor Inferior de Controle de Certeza, $V_{i c c}$, o Valor Superior de Controle da Contradição, $V_{s c c t}$, e o Valor Inferior de Controle da Contradição, $V_{i c c t}$, que indicam os valores máximos e mínimos aceitáveis no sistema para que determinada proposição seja considerada falsa ou verdadeira.

Após o tratamento das informações do LPA2v para remoção destes efeitos chega-se ao valor do chamado Grau de Certeza de Valor Real $\left(G_{C R}\right)$, que considera o valor de interpolação do reticulado em um estado lógico paraconsistente $\varepsilon \tau\left(G_{c}, G_{c t}\right)$ e o segmento de reta que vai do ponto máximo de $G_{c}$ (quando $G_{c}$ é positivo) ou ao valor mínimo de $G_{c}$ (quando este é positivo), segundo relação mostrada na Eq. (4).

A representação geométrica do $G_{C R}$ é mostrada na Fig. 2 .

$$
G_{C R}= \begin{cases}\left(1-\sqrt{\left(1-\left|G_{c}\right|\right)^{2}+G_{c t}^{2}}\right) & \text { se } G_{c}>0 \\ \left(\sqrt{\left(1-\left|G_{c}\right|\right)^{2}+G_{c t}^{2}}-1\right) & \text { se } G_{c}<0\end{cases}
$$

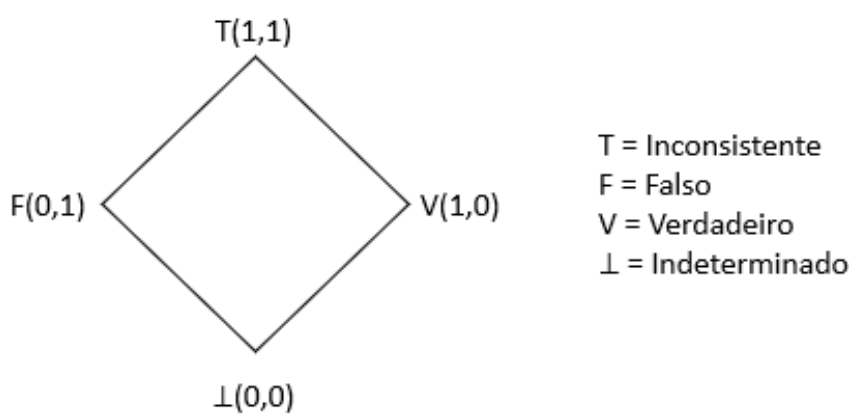

Figura 1: Representação do reticulado da LPA2v 


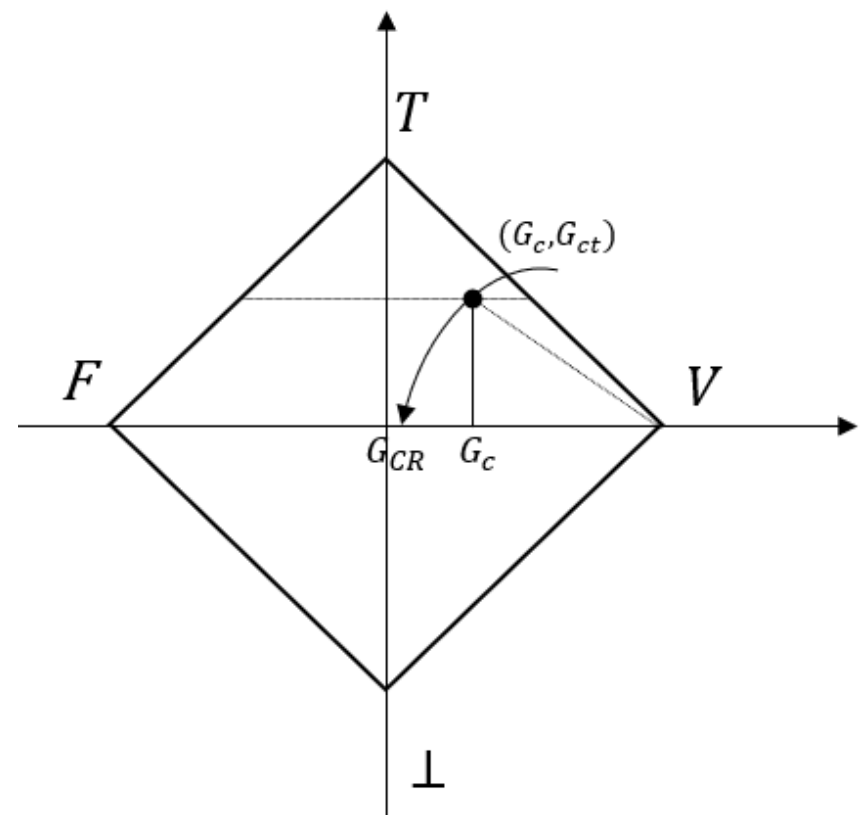

Figura 2: Representação geométrica do $G_{C R}$ na LPA2V para $G_{c}>0$

\subsection{Redes Neurais Artificiais Paraconsistentes}

Uma RNAP se baseia na teoria das RNAs clássicas, porém, apresenta características distintas - entre elas o fato de usar a LPA2v em sua construção e funcionamento (Lopes, 2009), e por utilizar diferentes tipos de unidades básicas - ao contrário de uma RNA que, normalmente, possui apenas um tipo de neurônio.

A unidade neural básica de uma RNAP é a chamada Célula Neural Artificial Paraconsistente (CNAP); esta pode ter diferentes configurações e utiliza uma equação estrutural básica (EEB) para equacionar sinais de entrada e convertêlos em um sinal de saída, transmitido para outras CNAPs.

Uma CNAP é formada por uma Célula Artificial Paraconsistente básica $(\mathrm{CAPb})$ modificada, caracterizada por receber dois sinais de entrada: um representando o valor de entrada $\mu$ da LPA2v, e o outro o valor $\lambda$; sua saída $\mu$ r, chamada de saída resultante, é calculada pelos valores de $G_{c}$ e $G_{c t}$ através da execução das análises da LPA2v.

Um esquema de funcionamento de uma $\mathrm{CAPb}$ é mostrado na Fig. 3, em que $\mu$ representa o grau de evidência favorável, $\lambda$ o grau de evidência desfavorável, $V_{s c c} 0$ valor superior de controle da certeza, $V_{\text {icc }}$ o valor inferior de controle da certeza, $V_{\text {scct }}$ o valor superior de controle da contradição, $V_{\text {icct }}$ o valor inferior de controle da contradição, $G_{c} 0$ grau de certeza, $G_{c t}$ o grau de contradição e o conjunto $V, F, I$ as saídas lógicas possíveis - Verdadeiro, Falso e Indeterminado.

A primeira modificação realizada em uma $\mathrm{CAPb}$ para que seja considerada uma CNAP é a normalização de sua saída, para garantir seu pertencimento ao intervalo real $[0,1]$, de acordo com a relação $\mu_{r}=\frac{(\mu-\lambda+1)}{2}$.

A segunda diferença em uma CAPb é a existência da "análise da tolerância", definida através de fatores de diferentes fatores de controle que podem ou não estar presen- tes e que auxiliam a equilibrar o processamento da CNAP no caso de insuficiência de informações ou por excesso de contradição nestas. Alguns fatores de tolerância em uma CNAP são: o fator de tolerância à contradição $\left(F T_{c t}\right)$, o fator de tolerância à certeza $\left(F T_{C}\right)$, o fator de tolerância à decisão $\left(F T_{d}\right)$ e o fator de aprendizagem $\left(F_{A}\right)$. Todos os valores externos variam no intervalo $[0,1]$ (Da Silva Filho et al., 2008).

De modo especial, o $F_{A}$ tem como função ajustar a rapidez com que uma CNAP aprenderá, variando conforme a seguinte classificação:

i. $F_{A}=0,0-$ não há aprendizado

ii. $F_{A}=0,5-$ aprendizado natural

iii. $F_{A}=1,0-$ função degrau

Ao executarem diferentes algoritmos paraconsistentes, especializados, CNAPs podem ser unidas para formarem sistemas neurais artificiais paraconsistentes (SNAPs) e RNAPs

\subsection{Imagens Digitais}

Imagens digitais são formadas a partir de uma correlação direta entre o número de fótons incidentes no sensor de uma câmera digital com o valor de intensidade dos pixels representados digitalmente, podendo-se estabelecer a relação geral $N \infty I$, descrita pela relação $\mathrm{N}=\mathrm{F} \Delta t \int q(\lambda) b(\lambda) d(\lambda)$, em que são relacionados os padrões de área do sensor $F$, o tempo de exposição $\Delta t$, a eficiência do equipamento $\mathrm{q}(\lambda)$ e o fluxo de fótons $\mathrm{b}(\lambda)$ incidentes,

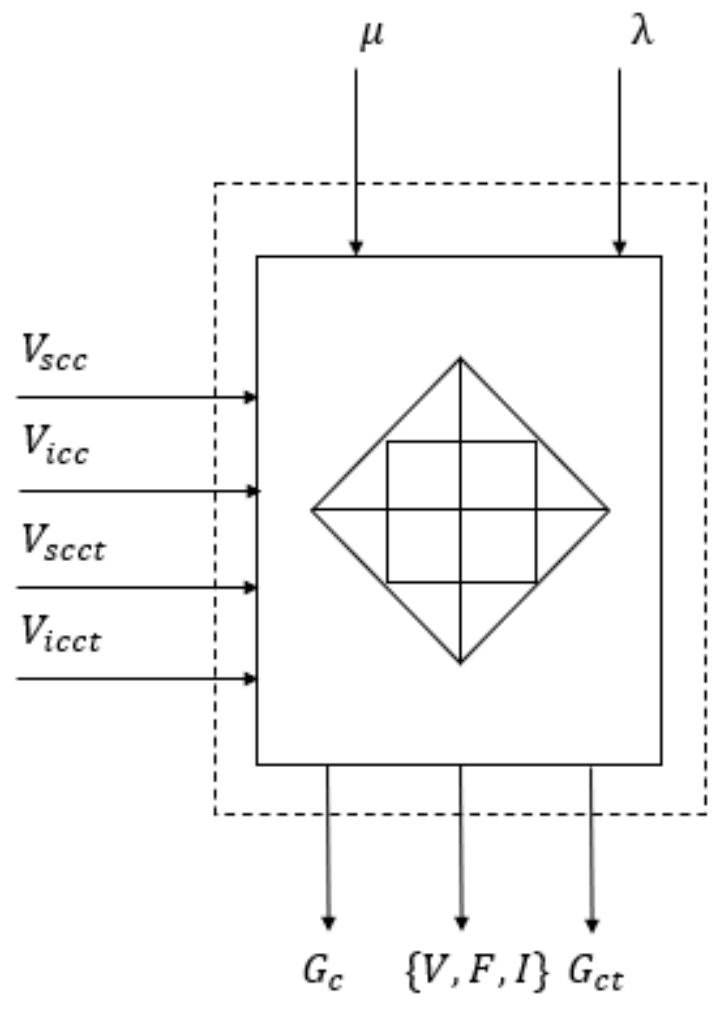

Figura 3: Esquema de funcionamento de uma $\mathrm{CAPb}$ 
sendo $\lambda$ o comprimento de onda específico para determinada cor.

Imagens são formadas a partir de níveis discretos e quantizados de intensidade em diferentes pixels (também chamados de "níveis de cinza"), cuja relação matemática válida é $g(i, j): B \mapsto G$ em que $B$ representa a matriz da imagem (em linhas e colunas) e $G$ as intensidades de nível de cinza sendo $G \in N$, no intervalo $[0,255]$.

Uma forma muito conveniente de se trabalhar com imagens digitais consiste em convertê-la exclusivamente para seus níveis de cinza, o que permite o trabalho com matrizes com um único canal de cor, tendo-se, então, uma matriz de trabalho de duas dimensões com valores no intervalo $[0,255]$, em que cada relação $g(i, j)$ para a linha $i$ e a coluna $j$ representa um pixel da imagem.

Imagens digitais podem ser transformadas a partir de três tipos diferentes de operadores: operadores globais, operadores pontuais, e operadores locais; estes, de interesse neste trabalho.

Operadores locais possuem aplicações extremamente importantes, como na redução de ruídos nas imagens, na aplicação de filtros de imagem ou no cálculo de gradientes.

Talvez o operador local mais conhecido seja o box filter, capaz de trocar o valor de intensidade dos pixels individualmente pela média aritmética dos valores das intensidades dos pixels adjacentes, definido, para uma matriz em 2 dimensões, pela relação $g(i, j)=\frac{1}{K L} \sum_{(k, l)} f(1-k, j-l)$, em que $K$ e $L$ representam as intensidades originais em cada pixel na horizontal e na vertical e $k$ e $l$ representam a quantidade de vizinhos considerados no cálculo.

A relação apresentada pode ser reescrita utilizando uma função-peso $w$, denominada kernel, assumindo a forma $g(i, j)=\sum_{k, l} w(l, k) f(1-k, j-l)$.

Equações que trabalham com kernels possuem a capacidade de trabalharem com combinações de diferentes valores nas funções-peso, chamados de operadores de filtro lineares; filtros no formato $g(i, j)=\sum_{(k, l)} f(1-k, j-l) w(l, k)$ com $g=f \times w$ são chamados de convoluções da função $f$ com kernel $w$.

O kernel do operador local box filter escrito como uma convolução é representado por $R_{3}^{(2)}=\frac{1}{9}\left[\begin{array}{lll}1 & 1 & 1 \\ 1 & 1 & 1 \\ 1 & 1 & 1\end{array}\right]$, em que $R$ representa o kernel, o valor subscrito o número de pixels vizinhos considerados, e o valor sobrescrito a dimensionalidade da matriz.

Outro operador local utilizado com o fim de suavizar imagens digitais através da utilização de um kernel específico é o Filtro Binomial (também chamado de Filtro Gaussiano). Seu funcionamento se baseia na diminuição os pesos relativos das intensidades individuais da imagem, aproximando-os de uma curva gaussiana, de forma $G(x, y)=\frac{1}{\left(2 \pi \sigma^{2}\right)} e^{\frac{-\left(x+y^{2}\right)}{2 \sigma^{2}}}$, em que $x$ e $y$ representam as dimensões verticais e $\sigma$ o desvio-padrão desejado a ser utilizado para a distribuição Gaussiana.

Para uma matriz em duas dimensões $\operatorname{com} \sigma \approx 0,85,0$ kernel utilizado é o representado por $R_{2}^{(3)}=\left[\begin{array}{lll}1 & 2 & 1 \\ 2 & 4 & 2 \\ 1 & 2 & 1\end{array}\right]$; seu efeito imediato na imagem é o surgimento de um borrão.

O cálculo da derivada primeira de um pixel em relação a seus vizinhos é de grande importância para a identificação de características da imagem como, por exemplo, a identificação de contornos - isto graças à diferença brusca de intensidade em pixels vizinhos. Isto é conseguido através do Filtro Gradiente, operador local definido pela aproximação da primeira derivada das funções representativas dos valores de intensidade, usado para a obtenção de propriedades das imagens em pontos isolados.

Para uma matriz unidimensional, a primeira derivada entre dois pontos adjacentes pode ser calculada por $f^{\prime}(i) \approx$ $\frac{\Delta f}{\Delta x}=\frac{f(i+1)-f(i)}{\Delta x}$, valendo a relação $\Delta f(i)=f(i+1)-f(i)$. Como $\Delta f$ representa uma operação de subtração, ele pode ser escrito na forma de um operador $\Delta f=\left[\begin{array}{c}1 \\ -1\end{array}\right] \mathrm{e}$ utilizado como uma convolução.

Para um espaço bidimensional o operador gradiente $\nabla$ consiste das derivadas parciais $\nabla\left[\begin{array}{c}\frac{\partial}{\partial x} \\ \frac{\partial}{\partial y}\end{array}\right] \approx\left[\begin{array}{c}\frac{\Delta x}{\Delta X} \\ \frac{\Delta y}{\Delta Y}\end{array}\right]$, sendo o gradiente da função calculado tanto para a $x$ como para $y$ tal que $\nabla g=\nabla \times g=\left[\begin{array}{l}g_{x} \\ g_{y}\end{array}\right]$, com a magnitude do gradiente definida pela relação $|\nabla g|=\sqrt{g_{x}^{2}+g_{y}^{2}}$ e a sua direção pela relação $\alpha=\arctan \left(\frac{g_{y}}{g_{x}}\right)=\arctan 2\left(g_{y}, g_{x}\right)$.

O cálculo dos gradientes pode ser aproximado pela utilização de determinados operadores, em convoluções, tais como o Operador Sobel - em uma janela 3×3 - que combina os resultados da aplicação de um Filtro Gaussiano com o Filtro Gradiente, ou através do Operador Scharr uma modificação do Operador Sobel que busca minimizar o erro angular médio ponderado quadrático no domínio de Fourier sob a condição de que os filtros resultantes sejam numericamente consistentes. Ele também é usado no cálculo das derivadas primeiras e assume as formas $\Delta x=\left[\begin{array}{ccc}3 & 0 & -3 \\ 10 & 0 & -10 \\ 3 & 0 & -3\end{array}\right] e \Delta y=\left[\begin{array}{ccc}3 & 10 & 3 \\ 0 & 0 & 0 \\ -3 & -10 & -3\end{array}\right]$ para cada direção.

\section{Materiais e Métodos}

\subsection{Preparação}

As fotografias utilizadas neste trabalho foram tiradas com um smartphone Apple iPhone 8 plus com câmera dupla com resolução de $12 \mathrm{MP}$, sensor Apple iSight Duo Camera(8) tipo CMOS BSI com abertura f/1.8, tamanho $1 / 3$, com tamanho do pixel igual a $1.20 \mu \mathrm{m}$ e flash Quad-LED, conforme especificações dadas pelo fabricante. As imagens foram armazenadas em formato comprimido JPG e transferidas para o computador.

Esta pesquisa trabalhou com dois objetos: uma peça quadrada, cor salmão, com superfície de dimensões 105 

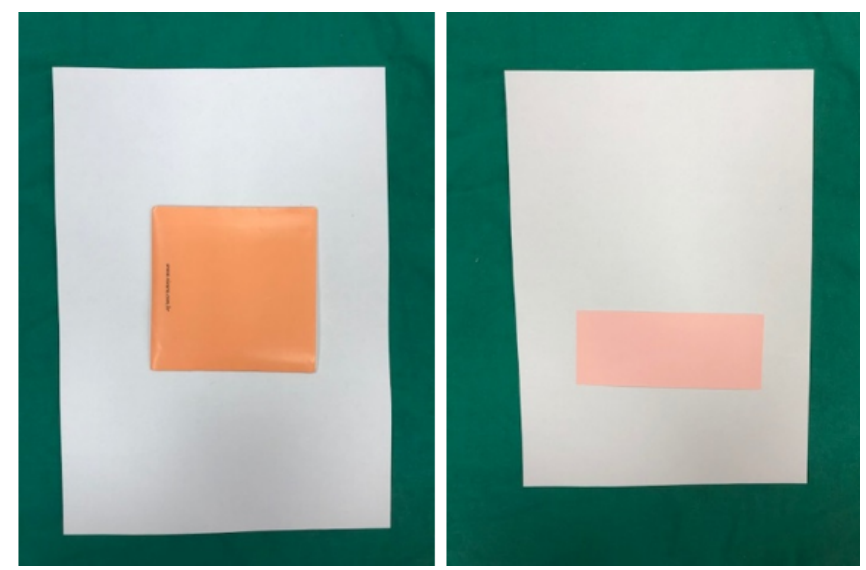

Figura 4: Exemplos de fotografias utilizando a peça quadrada e a peça retangular

mm x 105 mm e altura desprezível e com uma peça retangular, cor rosa, com superfície de dimensões $135 \mathrm{~mm}$ x 55 mm e altura desprezível.

Todas as dimensões informadas foram medidas com régua de alumínio. No momento da captura das imagens o flash ficou definido na opção "automático" e todos os objetos foram dispostos, individualmente, sobre um tecido verde - de forma a facilitar a separação do plano de fundo - e posicionados em cima de uma folha de papel tamanho A4, com dimensões nominais aferidas de 210 mm x 297 $\mathrm{mm}$.

A câmera foi posicionada em diferentes posições e sob diversas perspectivas dos objetos sem qualquer tipo de controle de posição, distância ou luminosidade, garantindo apenas que os objetos estivessem presentes na fotografia sem cortes ou obstáculos. Foram retiradas 25 fotografias de cada um dos objetos, perfazendo um total de 50 imagens. Exemplos das imagens utilizados são mostrados na Fig. 4

Os scripts para manipulação e coleta das propriedades das imagens assim como para a realização dos estudos estatísticos e execução das RNAPs foram desenvolvidos utilizando linguagem Python 3.8.5, considerando o padrão de Filtro de Bayer, utilizando as bibliotecas OpenCV 4.2.0 e NumPy 1.18.5. Todos foram executados em sistema operacional Linux na distribuição Ubuntu 20.04.1 LTS instalado em computador de uso pessoal com processador Intel Core i5-8265U CPU @ 1.600GHz 1.80 GHz e memória RAM de $8,00 \mathrm{~GB}$.

\subsection{Processamento Paraconsistente de Imagens}

O desenvolvimento da Unidade Paraconsistente de Convolução (UPC) foi inspirado pelo Controlador Paraconsistente Estatístico de Processos - CEP-LPA - descrito por Cruz (2015), cujo uso no cálculo da média aritmética de valores simulados por padrões gerados via software demonstrou ser eficaz.

AUPC foi composta por módulos extratores de evidência iniciais, com funções de preparar os dados de acordo com os kernels dos filtros aplicados - expressando-os como graus de evidência -, módulos extratores de evidência

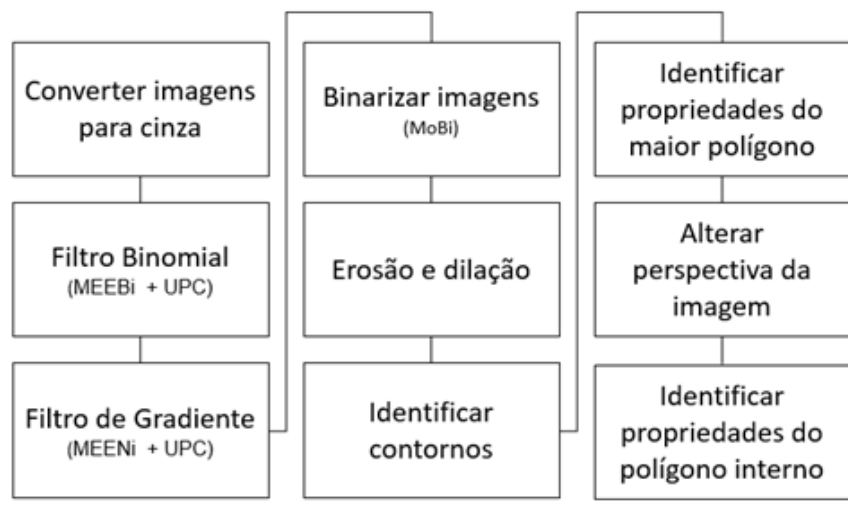

Figura 5: Fluxograma de processamento na UPC

finais, utilizados após cada iteração nos processos de convolução de forma a equalizar os valores processados sem incorrer em perda de precisão, e do Módulo de Convolução (MC), utilizado na realização das operações aritméticas do processo de convolução.

Ela contou também com módulos auxiliares, não envolvidos na convolução diretamente, mas utilizados por ela - como é o caso do Módulo de Binarização (MoBi). Um fluxograma contendo os passos para o processamento das imagens na UPC é mostrado na Fig. 5 e seus módulos são descritos a seguir.

\subsubsection{Módulo Extrator de Evidência Binomial inicial}

O Módulo Extrator de Evidência Binomial inicial (MEEBi) foi desenvolvido considerando um kernel de dimensões $3 \times 3$, em que o elemento central se alinha com o pixel de interesse em uma imagem.

A partir do kernel gaussiano e considerando todas as combinações de linha e coluna possíveis em uma matriz $3 \times 3$, o grau de evidência $\mu(i, j)$, em que $i$ representa a posição da linha do pixel no kernel $K$ de dimensões $m \times n$ em relação ao elemento-âncora e $j$ a posição da coluna, são calculados os valores dos pixels correspondentes a partir das relações mostradas nas Eqs. (5) a (13).

$$
\mu-1,-1=\left\{\begin{array}{l}
0,0625 \times \frac{K[i-1][j-1]}{255}, \text { se } i !=0 \text { e } j !=0 \\
\text { senão, zero }
\end{array}\right.
$$

$$
\begin{gathered}
\mu_{-1,0}=\left\{\begin{array}{l}
0,125 \times \frac{K[i-1][j]}{255}, \text { se i } !=0 \\
\text { senão, zero }
\end{array}\right. \\
\mu_{-1,1}=\left\{\begin{array}{l}
0,0625 \times \frac{K[i-1][j+1]}{255}, \text { se i } !=0 \text { e } j !=n-1 \\
\text { senão, zero }
\end{array}\right. \\
\mu_{0,-1}=\left\{\begin{array}{l}
0,125 \times \frac{K[i][j-1]}{255} \\
\text { senão, zero }
\end{array}, \text { se } j !=0\right.
\end{gathered}
$$




$$
\begin{aligned}
& \mu_{0,0}=\left\{0,25 \times \frac{K[i][j]}{255}\right. \\
& \mu_{0,1}=\left\{\begin{array}{l}
0,125 \times \frac{K[i][j+1]}{255}, \text { se } \mathrm{j} !=\mathrm{n}-1 \\
\text { senão, zero }
\end{array}\right. \\
& \mu_{1,-1}=\left\{\begin{array}{l}
0,0625 \times \frac{K[i+1][j-1]}{255}, \text { se i } 1=\mathrm{m}-1 \text { e } \mathrm{j} !=0 \\
\text { senão, zero }
\end{array}\right. \\
& \mu_{1,0}=\left\{\begin{array}{l}
0,125 \times \frac{K[i+1][j]}{255}, \text { se i } !=\mathrm{m}-1 \\
\text { senão, zero }
\end{array}\right. \\
& \mu_{1,1}=\left\{\begin{array}{l}
0,0625 \times \frac{K[i+1][j+1]}{255}, \text { se il=m-1 e j!=n-1 } \\
\text { senão, zero }
\end{array}\right.
\end{aligned}
$$

\subsubsection{Módulo Extrator de Evidência Nablaiana inicial}

O cálculo do Filtro Gradiente, na UPC, é realizado através do Módulo Extrator de Evidência Nablaiana inicial (MEENi), concebido utilizando um kernel de dimensões $3 \times 3$ tal como o MEEBi, utilizando uma modificação do Operador Scharr; isto, porque a LP define que os graus de evidência utilizados estejam dentro do intervalo $[0,1]$, condição não satisfeita na completude pelo operador em questão devido a existência de pesos negativos aí, que não seriam eliminados por uma normalização simples.

A partir do Operador Scharr e, considerando todas as combinações de linha e coluna possíveis em uma matriz $3 \times 3$, o grau de evidência $\mu(i, j)$, em que $i$ representa a posição da linha do pixel no kernel $K$ de dimensões $m x n$ em relação ao elemento-âncora e $j$ a posição da coluna, as evidências são calculadas a partir das relações mostradas nas Eqs. (14) a (22) que já consideram o cálculo nas dimensões $x$ e $y$.

$$
\begin{gathered}
\mu_{-1,-1}=\left\{\begin{array}{l}
0,21875 \times \frac{K[i-1][j-1]}{255}, \text { se } \mathrm{i} !=0 \text { e } j !=0 \\
\text { senão, zero }
\end{array}\right. \\
\mu_{-1,0}=\left\{\begin{array}{l}
0,31250 \times \frac{K[i-1][j]}{255}, \text { se } \mathrm{i} !=0 \\
\text { senão, zero }
\end{array}\right. \\
\mu_{-1,1}=\left\{\begin{array}{l}
0,40625 \times \frac{K[i-1][j+1]}{255}, \text { se i } \mathrm{i}=0 \text { e } \mathrm{j} !=\mathrm{n}-1 \\
\text { senão, zero }
\end{array}\right. \\
\mu_{0,-1}=\{\text { zero }
\end{gathered}
$$

$$
\begin{gathered}
\mu_{0,0}=\left\{0,31250 \times \frac{K[i][j]}{255}\right. \\
\mu_{0,1}=\left\{\begin{array}{l}
0,62500 \times \frac{K[i][j+1]}{255}, \text { se } j !=n-1 \\
\text { senão, zero }
\end{array}\right. \\
\mu_{1,-1}=\left\{\begin{array}{l}
0,21875 \times \frac{K[i+1][j-1]}{255}, \text { se } \mathrm{i} !=\mathrm{m}-1 \mathrm{e} \mathrm{j} !=0 \\
\text { senão, zero }
\end{array}\right. \\
\mu_{1,0}=\left\{\begin{array}{l}
0,31250 \times \frac{K[i+1][j]}{255}, \text { se } \mathrm{i} !=\mathrm{m}-1 \\
\text { senão, zero }
\end{array}\right. \\
\mu_{1,1}=\left\{\begin{array}{l}
0,40625 \times \frac{K[i+1][j+1]}{255}, \text { se i } \mathrm{i} !=\mathrm{m}-1 \mathrm{e} \mathrm{j} !=\mathrm{n}-1 \\
\text { senão, zero }
\end{array}\right.
\end{gathered}
$$

\subsubsection{Módulo de Binarização}

A imagem processada no MEENi ainda passa por processos sucessivos de binarização, de forma que apenas as arestas sejam mantidas na matriz representante. A primeira binarização, utilizando o MoBi considera um valores-limiares empíricos e ajustados conforme necessidade.

O MoBi utiliza uma função-degrau com o fim de binarizar a imagem - isto é, permitir apenas valores zero ou 255 em seus pixels. Para ele, foi definido um limiar de intensidade de pixel empírico, sendo válida a relação $g(i, j)=\left\{\begin{array}{l}\text { zero, se } g(i, j)<T \\ \text { senão, } 255\end{array}\right.$ em que $g(i, j)$ representa o valor de cinza na posição $(i, j)$ em que $i$ representa a linha, $j$ a coluna e $T$ representa o valor-limiar utilizado como referência no degrau, apresentados na equação.

A imagem binarizada é limpa através de processos de erosão e dilação, próprios da biblioteca OpenCV.

\subsubsection{Módulos Extratores de Evidência}

Os Módulos Extratores de Evidência intermediária (MEEi) e final (MEEf) são módulos utilizados no ajuste dos graus de evidência durante seus cálculos no módulo de convolução, de forma a garantir não apenas sua precisão como, ainda, para realizar o ajuste de intensidade dos pixels, diminuída nos módulos extratores de evidência.

O MEEi define uma função linear $f(\mu)$ tal que $f(\mu)=2 \times \mu$, enquanto o MEEf define uma função linear $f(\mu)$ tal que $f(\mu)=255 \times \mu$.

\subsubsection{Módulo de Convolução}

O Módulo de Convolução (MC) é o módulo desenvolvido com a função de executar a operação de convolução nas imagens, definida por $V=\left|\frac{\sum_{i=i}\left(\sum_{i=j} f_{i j} d_{i j}\right)}{F}\right|$ em que $V$ representa o valor do pixel-âncora, $f_{i j}$ o coeficiente do kernel de convolução na posição $(i, j), d_{i j}$ o dado encontrado no pixel correspondente a $f_{i j}$ e $F$ a soma dos coeficientes do kernel. 


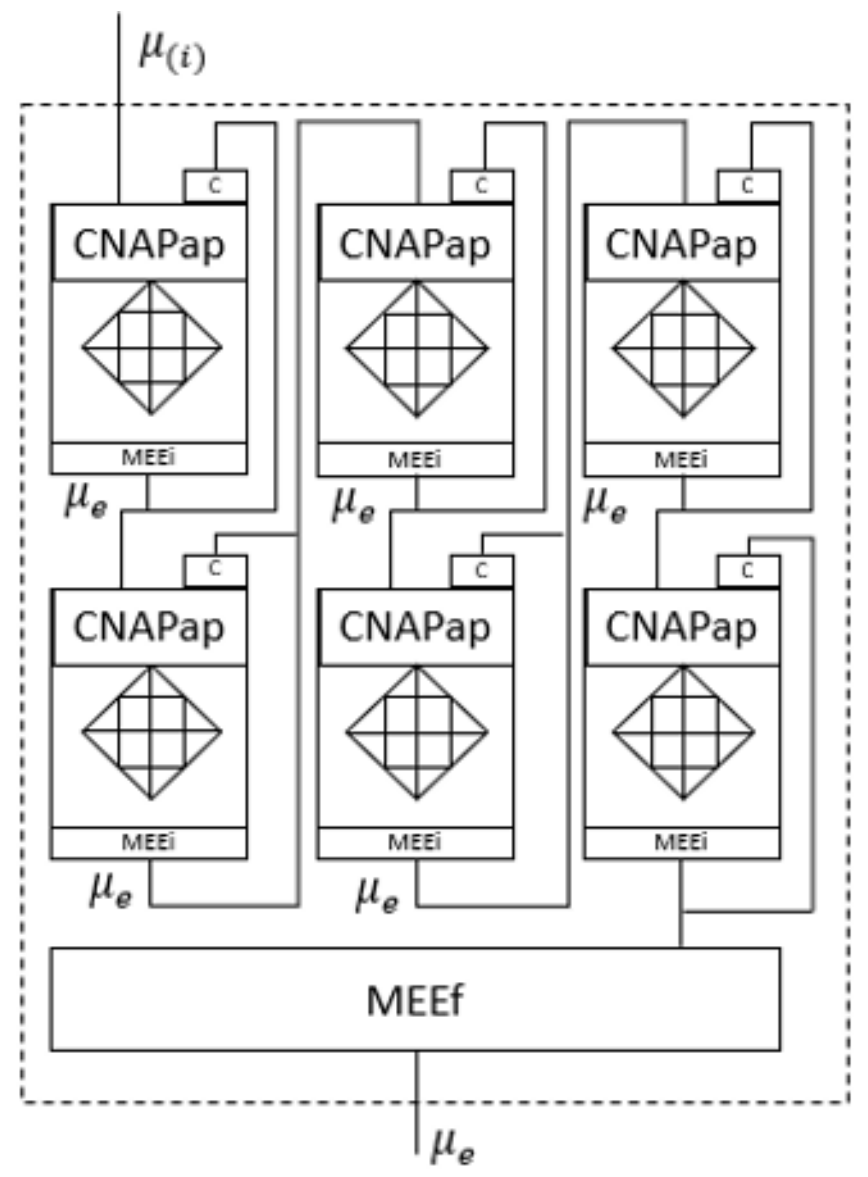

Figura 6: Esquema do funcionamento da UC com 6 CNAPap ligadas em série

Uma vez que os valores do kernel estejam disponíveis, por terem sido calculados pelos módulos extratores através de $f_{i j}$ e $d_{i j}$, e o valor de $F$ já tenha sido definido durante as extrações, os valores de intensidade para cada âncora podem ser calculados individualmente através das somatórias em $(i, j)$.

Sua base é uma CNAPap, capaz de aprender valores reais no intervalo fechado $[0,1]$, com $F A=1$, retornando um grau de evidência resultante na saída calculado conforme a Eq. (23), em que $\mu_{i}$ representa o grau de evidência favorável apresentado na entrada e $\mu_{E(i-1)}$ o grau de evidência resultante anterior.

$$
\mu_{E(i)}=\frac{\mu_{i}-\left(1-\left(\mu_{E(i-1)}\right)+1\right.}{2}
$$

Foram utilizadas 6 CNAPaps ligadas em série, incluída uma instância do MEEi entre elas; isto de forma a resgatar o valor às somatórias. Ao final, a evidência calculada foi convertida novamente ao valor de intensidade por meio do MEEf. Um esquema de funcionamento é mostrado na Fig. 6

\subsection{Etapas do processamento}

As cópias das imagens, armazenadas em pastas segregadas, seguiram o fluxo de processamento via código, que incluiu suas conversões para a escala de cinza - considerando o código de conversão de cores COLOR_BGR2GRAY, do OpenCV. As imagens não foram redimensionadas de forma a preservar suas escalas originais.

Como estratégia geral, o primeiro objetivo a ser atingido foi o de se encontrar a folha de papel $\mathrm{A}_{4}$ na imagem sendo ele "o maior contorno" presente na fotografia - e considerar apenas sua área no processamento das propriedades.

Uma vez que o tamanho da folha de papel é conhecido, isto possibilitou ao algoritmo calcular as dimensões dos demais objetos, cujos valores são proporcionais aos da folha A4.

O primeiro passo do processamento na UPC foi a aplicação do filtro binomial, combinando a utilização tanto do MEEBi como da UC, o que proveu uma imagem suavizada, com menor magnitude de ruído.

Uma vez aplicado o Filtro Gaussiano, isto permitiu a utilização do MEENi juntamente com a UC, em um processo subsequente, capaz de identificar os contornos presentes na imagem através das diferenças abruptas de intensidades encontradas nos pixels vizinhos da fotografia.

A imagem resultante foi binarizada pela aplicação do $\mathrm{MoBi}$; ações de dilação e erosão disponíveis no OpenCV foram aplicadas, então, de forma a remover os chamados outliers, isto é, pixels próximos às extremidades com intensidades muito altas ou muito baixas presentes em regiões em que não seriam esperados (uma vez que seu entorno seja, justamente, o contrário).

As informações sobre os contornos existentes nas imagens foram extraídas utilizando métodos próprios da linguagem Python e/ou do OpenCV entendidos como "uma curva que une todos os pontos contínuos (ao longo da fronteira), tendo a mesma cor ou intensidade".

Neste caso, o contorno de interesse é o mais externo, portanto as propriedades foram retiradas utilizando a hierarquia RETR_EXTERNAL, sob modo de aproximação CHAN_APPROX_SIMPLE, que armazena apenas os pontos das 4 coordenadas das arestas em memória, o suficiente para reconstruir, virtualmente, os objetos.

As arestas foram reconstruídas via código e as informações sobre a área e perímetro do maior polígono foram encontradas através dos métodos contourArea() e arcLength(), respectivamente.

Tendo o maior contorno sido identificado, representando a folha $\mathrm{A}_{4}$, a imagem foi ajustada via código de forma a mudar sua perspectiva, ignorando a existência do conteúdo externo (o plano de fundo, verde) e considerando apenas a área do papel como válida para a imagem. Uma vez que as dimensões da folha se tornaram conhecidas, os dados de medições dos polígonos internos puderam ser determinados proporcionalmente, realizando novamente as operações de determinação de perímetros e áreas dos polígonos internos - agora, os únicos presentes na imagem. 


\subsection{Classificação dos Objetos}

Um problema de classificação típico é definido para um conjunto $\Omega$ composto por um número $K$ de classes $c$ tal que $\Omega=\omega 1$... $\omega K$. Nele, assume-se a existência de uma característica e para a qual existe uma função $f$ capaz de determinar a que classe determinado dado pertence, tal que $c=f(e)$ e $\operatorname{com} c \in \Omega$.

A CNAP utilizada foi construída considerando um fator de aprendizagem $F A=1$, fator de tolerância à contradição $F t_{c t}=1$, fator de tolerância à certeza $F t_{c}=1$, fator de tolerância à decisão $F t_{D}=1$ e fator de aprendizagem $F A=1$.

Ela recebeu como graus de evidência $\mu$ os valores de área e de perímetro das imagens, normalizados de acordo com as Eqs. (24) e (25) de forma a mantê-los no intervalo $[0,1]$, definido pela LPA2v, em que $V$ representa o valor normalizado, $A$ representa o valor da área, $P$ o valor do perímetro e $L$ o maior lado encontrado para os polígonos.

$$
\begin{gathered}
V=\frac{\sqrt{A}}{L} \\
V=0,25 \times \frac{P}{L}
\end{gathered}
$$

Os graus de evidência normalizados foram processados através da execução do Algoritmo da Célula Neural Artificial Paraconsistente padrão,definindo uma saída resultante, $\mu r$, também é normalizada, responsável pela classificação geométrica da figura.

$O$ resultado calculado pela $\mu$ r determinou a classificação dos objetos, sendo um valor de $\mu_{r} \geq 0,71$ classificado como um "quadrado" e um valor de $\mu_{r}<0,71$ classificado como "retângulo".

\subsection{Detecção de Desvios}

O módulo de detecção do desvio da média tem a função de, a partir da classificação dada pela CNAP de classificação de objetos, identificar se as dimensões obtidas da imagem para o objeto em questão estão de acordo com o esperado - o que, em uma linha de produção, por exemplo, tem importante função na identificação de desvios de processo e/ou de materiais com defeitos, podendo serem removidos de uma esteira.

A detecção de valores fora dos parâmetros de controle foi conseguida através da execução do Algoritmo da Célula Neural Artificial Paraconsistente de detecção de igualdade (CNAPdi), realizado pela comparação entre o valor informado para o Fator de tolerância à contradição $F t_{c t}$ e o valor resultante do processamento dos graus de evidência $\mu_{1} \mathrm{e}$ $\mu_{2}$ informados na entrada. Uma saída $S_{1}$ com valor lógico Verdadeiro indica que os sinais são iguais; já a saída $S_{1}$ com valor lógico Falso indica uma desigualdade.

A sensibilidade do sistema foi controlada pelo valor definido para o $F t_{c t}$, podendo ser maior ou menor de acordo com a tolerância aos desvios definida pelos processos de qualidade envolvidos. Neste projeto utilizou-se um valor de $F t_{c t}=0,001$, considerando um processo bastante estrito com as dimensões dos objetos fabricados.
A CNAP utilizada recebeu como valor de $\mu_{1}$ o valor da área normalizado, enquanto $\mu_{2}$ apresentou valor fixo igual ao valor da área normalizado nominal para o quadrado ou o valor da área normalizado nominal para o retângulo.

\section{Resultados e Discussão}

As 50 imagens capturadas foram inicialmente varridas visualmente para identificação de possíveis interferências. Nesta etapa foram removidas as imagens cuja utilização do flash acabou sendo refletida na superfície dos objetos e que, por isso, criaram marcas na fotografia que pudessem causar erros no processamento subsequente, assim como fotografias nas quais a sombra também criou efeitos indesejados. Em um único caso a imagem foi descartada por apresentar inconsistências no plano de fundo.

\subsection{Análise dos histogramas de frequências dos níveis de cinza e cálculo da variância}

A análise dos histogramas de frequências dos níveis de cinza, extraídos pela biblioteca OpenCV demonstrou uma tendência de comportamento semelhante independente do objeto utilizado - quadrado ou retangular. Em ambos existiu uma concentração entre as intensidades entre 50 e 80 , aproximadamente e, logo após, entre 170 e 230.

O cálculo das variâncias das imagens também revelou algumas tendências: enquanto o objeto quadrado apresentou valor de variância médio de 5215,9410 $\pm 339,4528$, o objeto retangular apresentou valor médio de 5820,9890 土 381,0599 - uma coesão um pouco menor, porém com valor médio de variância das intensidades significativamente superior, o que visualmente significa um contraste mais acentuado para a imagem retangular do que para o quadrado.

Isto pode ser explicado pelo fato de, no geral, a diferença entre pixels claros e escuros ser mais acentuada para o retângulo do que no quadrado; isto, porque o quadrado, cujas intensidades são escuras, parecidas com as do plano de fundo, e cuja área é maior que a do objeto retangular, acaba diminuindo os efeitos do papel $\mathrm{A}_{4}$, branco, na contribuição para baixos níveis de intensidade, na imagem.

\subsection{Aplicação do Filtro Binomial}

O filtro binomial aplicado através da utilização do MEEBi em conjunto com a UPC apresentou resultados visuais muito semelhantes aos resultados encontrados pela utilização do mesmo filtro, porém, via biblioteca OpenCV considerando um desvio-padrão $\sigma=0,85$ e um kernel do tipo 3x3. A comparação é mostrada na Fig. 7.

O efeito imediato da aplicação do Filtro Binomial é a presença de um borrão bastante discreto, acentuado em algumas regiões, principalmente nas quais se observa contraste de intensidades.

Apesar de os resultados visuais serem muito similares, o tempo de execução, calculado pela biblioteca Time da linguagem Python, é aqui um fator que merece destaque: enquanto a aplicação do Filtro Gaussiano via biblioteca demorou uma média de 0,014 \pm 0,005 segundos (medidos 

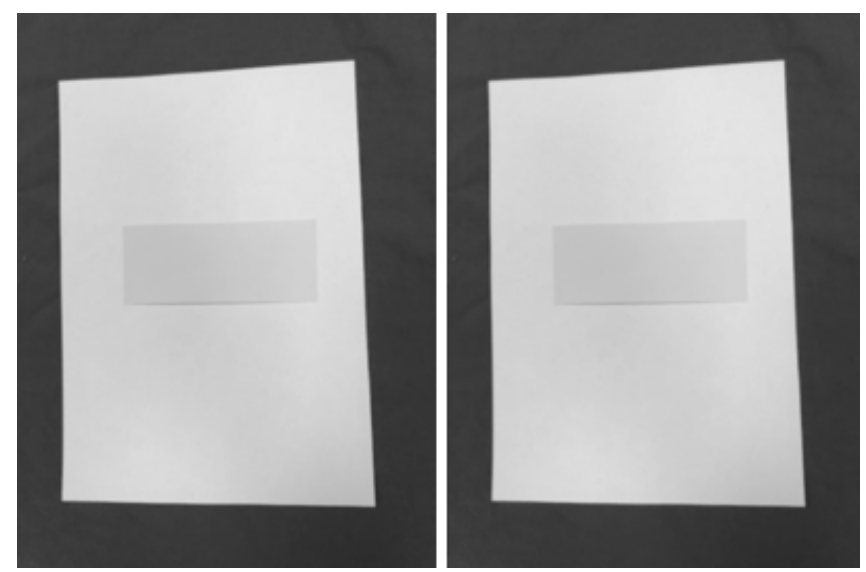

Figura 7: Comparação entre imagens após aplicação do Filtro Binomial (utilizando biblioteca-padrão, à esquerda e via MEEBi + MC, à direita)

para 10 amostras aleatórias) para ser executado, o processamento via MEEBi + MC levou uma média de 421,593 \pm 27,569 segundos para as mesmas amostras, valor mais de 30.000 vezes superior.

Aqui, a forma com que o algoritmo foi construído tem importante influência, uma vez que os passos foram dados sequencialmente, pixel a pixel, fator que pode ser trabalhado.

A comparação visual dos histogramas de frequências de níveis de cinza para uma figura aleatória antes (Fig. 8) e após aplicação do Filtro Binomial via MEEBi + MC (Fig. 9) evidencia uma suavização da distribuição de frequências dos níveis de cinza conforme esperado; este efeito é bastante evidente, por exemplo, quando se observam as frequências das intensidades entre 50 e 70.

A imagem utilizada para a geração do histograma apresentado possui um valor de variância determinado diretamente pela biblioteca NumPy igual a 5704,79.

Utilizando a mesma biblioteca, porém, determinando as variâncias das imagens processadas pelo Filtro Binomial

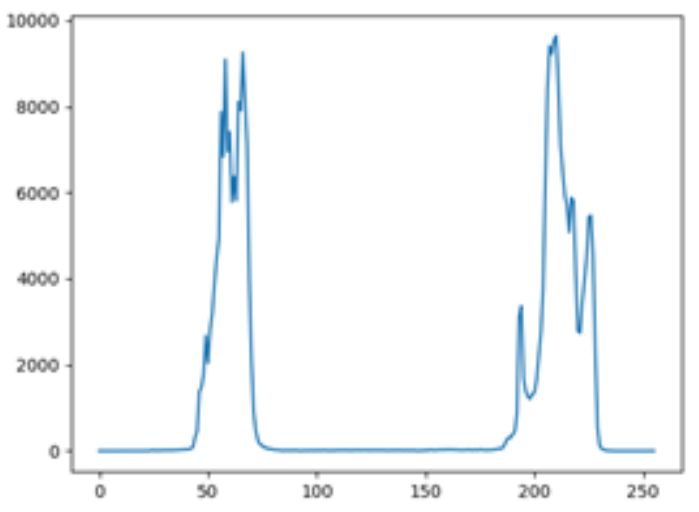

Figura 8: Histograma de intensidades de cinza encontrado para a figura aleatória

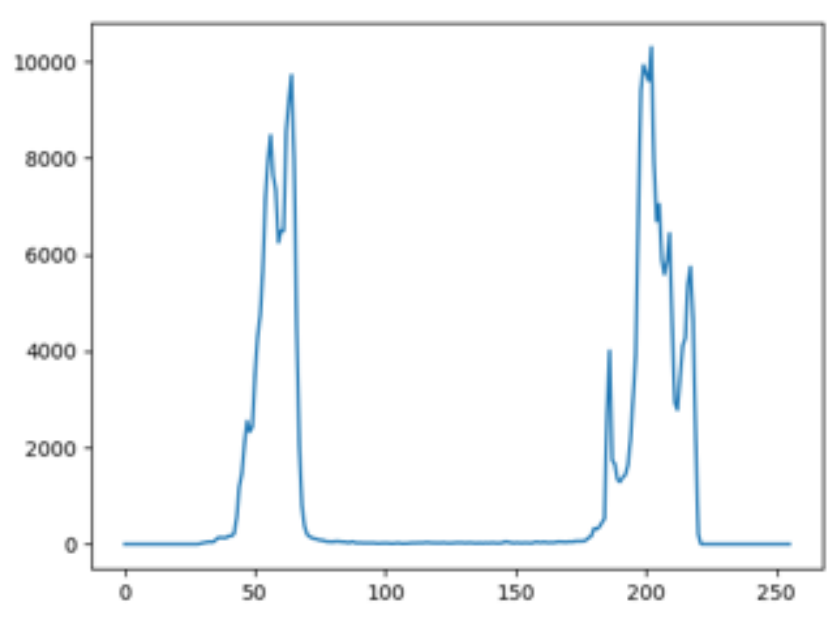

Figura 9: Histogramas de intensidades de cinza encontrado para a figura após aplicação do Filtro Gaussiano - via MEEBi + MC

via MEEBi + MC e via biblioteca OpenCV, os números são respectivamente de 5241,543 e 5665,9 - uma diminuição na variância da imagem de 8,12\% utilizando o MEEBi + $\mathrm{MC}$, contra $0,6 \%$ via biblioteca, o que ilustra, neste caso, uma boa vantagem da utilização da UPC em detrimento da biblioteca de larga utilização.

\subsection{Aplicação do Filtro Gradiente, binarização e identificação de contornos}

A realização da convolução através da MEENi +UPC foi realizada conforme fluxo apresentado anteriormente.

Uma diferença importante em comparação do processo paraconsistente com o uso da biblioteca OpenCV diz respeito à realização posterior de operações de binarização, de forma a isolar os contornos da imagem - sendo, portanto, uma operação realizada em dois passos distintos.

A execução da MEENi (antes da utilização do MoBi como auxiliar) mostrou-se bastante sensível à presença de imperfeições na imagem, como ilustrado na Fig. 10, em que a região com reflexo do flash ficou bem evidente (ainda que na imagem original este reflexo fosse visualmente desprezível).

A aplicação da MoBi mostrou-se um processo extremamente crítico, tendo em vista as diferentes condições sob as quais as fotografias foram tiradas influírem nas intensidades dos pixels presentes na imagem; assim, os limiares precisaram ser ajustados empiricamente, tendo os melhores resultados visuais sido encontrados para $T=220$ para a primeira iteração, e $T=255$ para a segunda.

Processos de dilação e erosão foram feitos em cima da imagem processada via MEENi + UPC, após binarização, com fins de diminuir os ruídos encontrados.

Um exemplo de imagem resultante é mostrada na Fig. 11, em que os contornos da folha $\mathrm{A} / 4$ e do objeto quadrado são claramente identificados. 

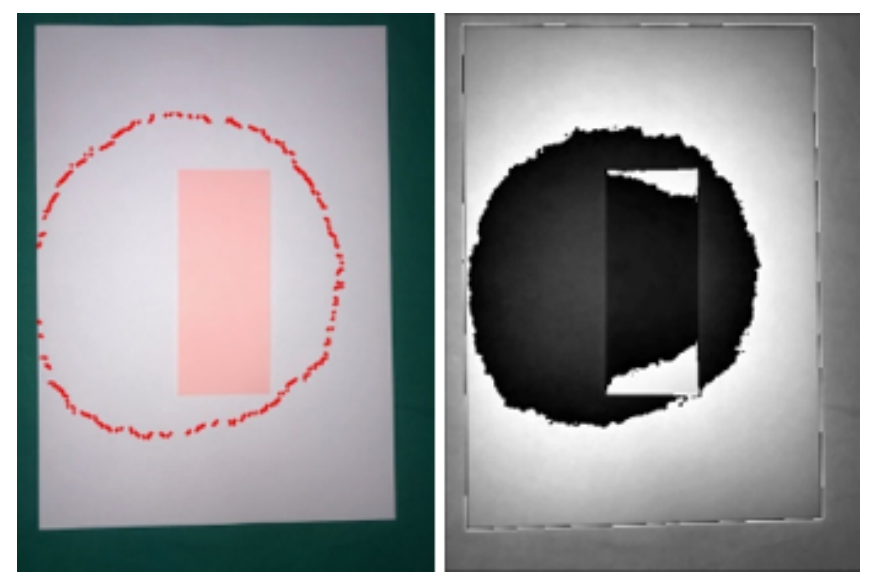

Figura 10: Exemplo de sensibilidade da MEENi ao reflexo do flash

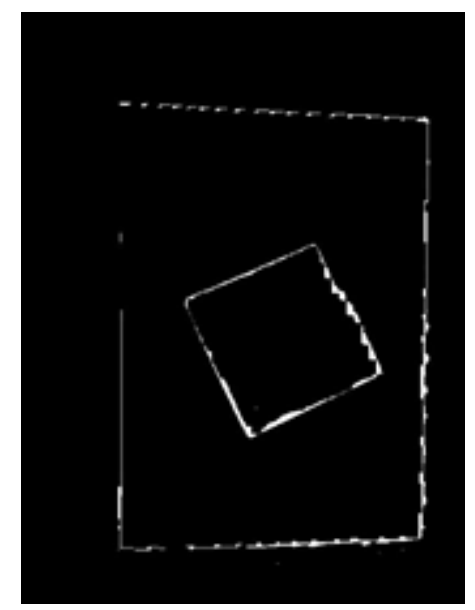

Figura 11: Extração dos contornos via MEENi + UPC + MoBi

\subsection{Extração das propriedades}

A etapa de extração das propriedades das imagens englobou as ações de identificação dos polígonos presentes na fotografia através de aproximações disponíveis pela biblioteca OpenCV, a ordenação por área (de forma a se identificar o maior de todos - no caso, a folha A4), a mudança de perspectiva da imagem via script em linguagem Python, uma nova identificação dos polígonos, agora buscando encontrar o objeto quadrado ou o objeto retangular, e a extração das propriedades de perímetro e área.

A partir da imagem tratada pela MEENi + UPC + MoBi e da execução das funções de dilação e erosão foram extraídos os contornos presentes na imagem, via código, considerando apenas os pontos mais extremos.

A manipulação das matrizes contendo as posições dos contornos externos permitiu identificá-los ao redor da folha de papel, indicando a presença de uma aresta.

A matriz contendo os pontos do contorno externo foi considerada para a realização de uma transformação, que ajustou o conteúdo interno deste polígono à tela, remo- vendo os elementos externos e reajustando a proporção na tela dos elementos internos, o que permitiu uma nova descoberta de contornos e posterior indicação na figura.

A mudança de perspectiva da imagem (com retirada do fundo verde) e a identificação dos contornos do objeto interno são ilustrados na Fig. 12.

O modo de extração das medidas dos objetos foi baseado no trabalho de Mário et al. (2012) em que, com o auxílio da LP, foram estimadas as alturas de árvores em fotografias, com a premissa da presença de um objeto de referência de dimensões conhecidas posicionado próximo a elas. Neste trabalho, o objeto de dimensões conhecidas é a folha A4, e são descritas pela altura e pela largura da própria imagem.

As distâncias entre os pontos mais externos foram calculadas a partir da obtenção dos dados dos vértices, permitindo a estimativa de suas dimensões reais considerando a proporção de distância da folha de papel, tal como mostrado na Fig. 13.

A distribuição dos valores encontrados para o lado do objeto quadrado apresentou valor médio de 104,84 $\pm 1,96$ $\mathrm{mm}$, um erro de $0,1 \%$ considerando o valor nominal me-

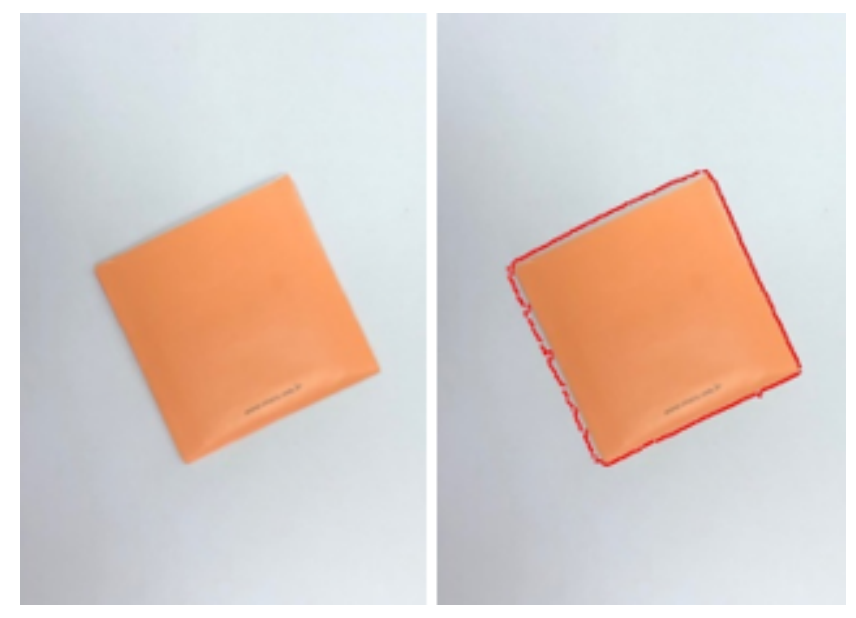

Figura 12: Transformação de perspectiva e determinação de contornos

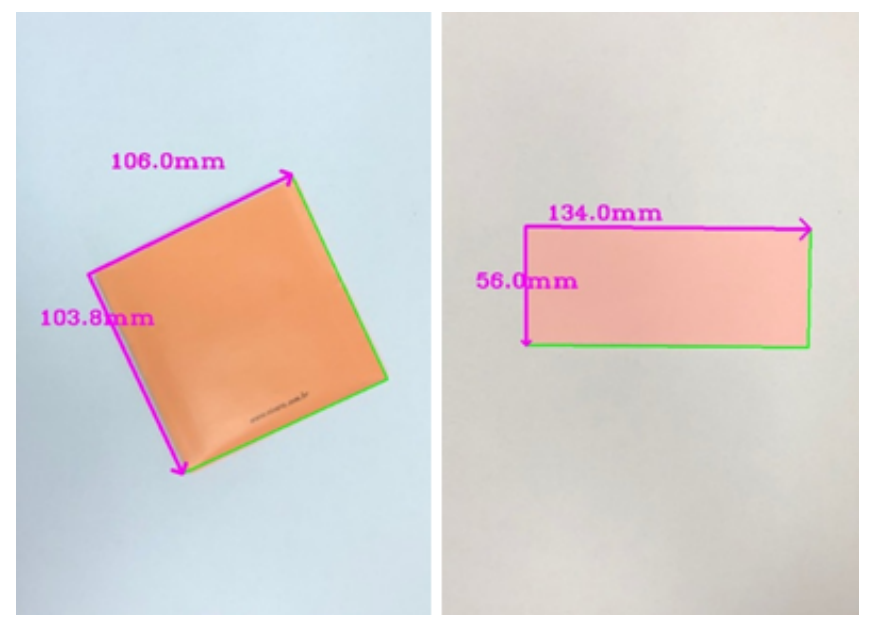

Figura 13: Exemplo de dimensões obtidas para os objetos 
dido pela régua de alumínio; já sua mediana apresentou o valor exato de 105,0 $\mathrm{mm}$.

A área apresentou valor médio calculado de 10989,82 \pm $190,75 \mathrm{~mm}^{2}$, um erro de 0,32\% em relação à área teórica (de $11025,00 \mathrm{~mm}^{2}$ ) e o perímetro apresentou valor de 419,37 \pm $3,64 \mathrm{~mm}$, um erro de $0,15 \%$ em relação ao valor esperado (de 420,00 mm).

No caso do objeto retangular, a distribuição dos valores encontrados para a base apresentou valor médio de 133,58 $\pm 0,97 \mathrm{~mm}$ - um erro de $1,04 \%$ em relação ao valor nominal - enquanto a altura apresentou valor médio de 54,6 $6 \pm 0,9$ $\mathrm{mm}$ - um erro de $0,72 \%$ em relação ao valor teórico; as medianas para a base e a altura encontradas foram de, respectivamente, $134,0 \mathrm{~mm}$ e $55,0 \mathrm{~mm}$.

A área média do objeto retangular possui valor médio calculado de $7293,83 \pm 136,15 \mathrm{~mm}^{2}$, um erro de $1,77 \% \mathrm{em}$ comparação à área teórica (de $7425,00 \mathrm{~mm}^{2}$ ), e o perímetro apresentou valor médio de $376,37 \pm 2,75 \mathrm{~mm}$ - erro de 0,95\% em comparação com o valor esperado (de 380,00 $\mathrm{mm})$.

\subsection{Classificação dos objetos}

Os graus de evidência utilizados na CNAPp de classificação foram extraídos conforme descrição das Eqs. (24) e (25), sendo considerado o valor $L=135 \mathrm{~mm}$, como o maior lado entre os polígonos, referindo-se à base do objeto retangular.

Foram retiradas 15 amostras aleatórias entre os resultados esperados. Delas, 10 foram utilizadas como conjunto de treinamento da CNAPp, de forma a se estabelecer o melhor limiar utilizado na classificação, e 5 foram utilizadas para teste.

Os resultados da execução do algoritmo da célula neural artificial paraconsistente padrão são mostrados na Tabela 1.

A análise dos graus de evidência reais $\mu_{r}$ permite concluir que o objeto quadrado possui uma tendência a ter valores acima de 0,75 enquanto o objeto retangular tem a tendência de mostrar valores abaixo de 0,70. Ao estabelecer um limiar de classificação tal que $\mu_{r} \geq 0,71$ representa um objeto quadrado e um $\mu \mathrm{r}<0,71$ representando um objeto retangular, os resultados mostrados na Tabela 2 são encontrados para a classificação das imagens de teste.

A criação de uma matriz de confusão baseada nos resultados de classificação do conjunto de testes pela CNAPp

Tabela 1: Graus de evidência para os objetos (conjunto de treinamento)

\begin{tabular}{c|c|c|c}
$\mu_{1}$ & $\mu_{2}$ & Forma & $\mu_{\mathrm{r}}$ \\
\hline 0,770701 & 0,770741 & Quadrado & 0,770721 \\
0,776994 & 0,777037 & Quadrado & 0,777015 \\
0,770741 & 0,770741 & Quadrado & 0,770741 \\
0,778116 & 0,778148 & Quadrado & 0,778132 \\
0,766822 & 0,767037 & Quadrado & 0,766929 \\
0,63115 & 0,69259 & Retângulo & 0,66048 \\
0,63011 & 0,6963 & Retângulo & 0,66158 \\
0,63354 & 0,6963 & Retângulo & 0,66345 \\
0,64167 & 0,7037 & Retângulo & 0,67122 \\
0,63829 & 0,7037 & Retângulo & 0,66937
\end{tabular}

Tabela 2: Resultados encontrados nos conjuntos de teste para a classificação da forma geométrica via CNAPp

\begin{tabular}{c|c|c|c|c}
$\mu_{1}$ & $\mu_{2}$ & Forma & $\mu_{r}$ & Resultado \\
\hline 0,782508 & 0,782593 & Quadrado & 0,78255 & Quadrado \\
0,770954 & 0,771111 & Quadrado & 0,771032 & Quadrado \\
0,790877 & 0,791111 & Quadrado & 0,790994 & Quadrado \\
0,629525 & 0,695926 & Retângulo & 0,661095 & Retângulo \\
0,623906 & 0,69037 & Retângulo & 0,655531 & Retângulo
\end{tabular}

apresenta os valores de acurácia, precisão, recall e $f$-score, todos iguais a 1 - valor máximo possível - indicativos de uma grande capacidade de realização da classificação de forma correta.

\subsection{Identificação de desvios}

O objetivo da utilização da CNAPdi no sistema proposto foi o de atuar como agente capaz de identificar desvios muito acentuados nas propriedades dos objetos em relação aos valores nominais, esperados, para cada categoria quadrado ou retângulo.

Diferentemente da CNAPp utilizada na classificação dos objetos, o objetivo do processamento dos graus de evidência, aqui, não foi o de se determinar um limiar na saída ótimo a partir do qual um desvio é ou não caracterizado, mas trabalhar em sua sensibilidade - isto, através do ajuste do $F t_{c t}$ ótimo, o que é variável sob diferentes cenários e valores de erros aceitáveis.

Considerando o valor nominal de área para o quadrado igual a $11025 \mathrm{~mm}^{2}$ e um fator de tolerância a contradição de $F t_{c t}=0,1$, por exemplo, a CNAPdi indicaria um desvio quando um objeto quadrado apresentasse área menor ou igual a $8122,7 \mathrm{~mm}^{2}$ (lado $90,12 \mathrm{~mm}$ ) ou maior ou igual a $14049,26 \mathrm{~mm}^{2}$ (lado 118,53 mm) - um erro de cerca de $15 \%$ em ambos os casos; a utilização de um $F t_{c t}=0,05$, por sua vez, seria capaz de manter os desvios entre uma área menor ou igual a $9650,99 \mathrm{~mm}^{2}$ (lado $98,23 \mathrm{~mm}$ ) ou maior ou igual a $12485,71 \mathrm{~mm}^{2}$ (lado $111,73 \mathrm{~mm}$ ) - neste caso, um erro de cerca de $6,5 \%$.

A Fig. 14 mostra um objeto quadrado, criado através de uma modificação do objeto original, para validar a capacidade da CNAPdi. Ele possui lado nominal igual a $104 \mathrm{~mm}-$ apenas $1 \mathrm{~mm}$ menor do que o quadrado original (sugerindo um erro de processo de 0,95\%) conforme medições com a régua de alumínio.

Extraídas suas propriedades via processamento, um lado $L_{1}$ foi determinado com tamanho $L_{1}=104,0 \mathrm{~mm}$ e o outro, $L_{2}$, com valor $L_{2}=104,1 \mathrm{~mm}$. Sua área A foi calculada como $A=10826,4 \mathrm{~mm}^{2}$ e seu perímetro $P=$ 416, $2 \mathrm{~mm}$.

A classificação feita pela CNAPp, com graus de evidência já normalizados $\mu_{1}=0,77074 \mathrm{e} \mu_{2}=0,770740$ categorizou corretamente como um quadrado.

Ao chegar na CNAPdi, contudo, e considerando um valor de tolerância à contradição $F t_{c t}=0,001$ e um valor de $\mu_{2}=0,77777$ (correspondente ao valor normalizado para a área nominal de $11025,0 \mathrm{~mm}^{2}$ ), o valor de $\mu_{\text {ctr }}$ é calculado como $\mu_{c t r}=0,4965$, inferior ao valor de $V_{\text {icct }}=0,49925$ determinado pelo $F t_{c t}$ indicando, aqui, um desvio nãoaceitável para o processo, o que seria capaz de disparar 


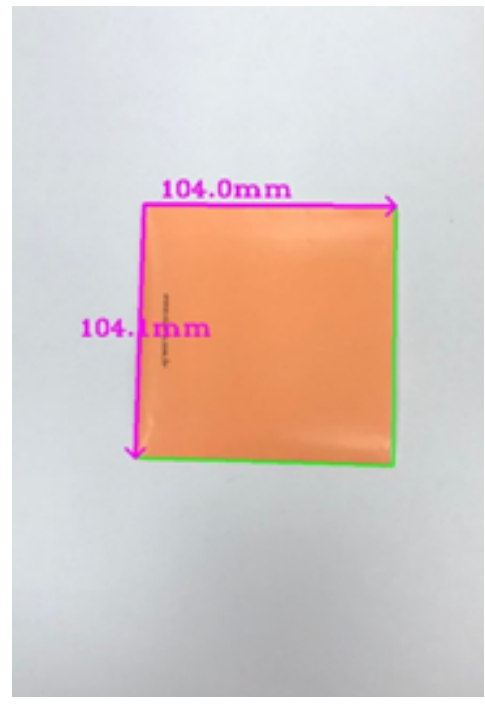

Figura 14: Objeto quadrado modificado utilizado no teste da CNAPdi

um alerta no processo - neste caso, um limite bastante crítico, de apenas $1 \mathrm{~mm}$.

\subsection{Discussão}

O uso de métodos ópticos em controle de qualidade vem sendo bastante difundido nos últimos anos, principalmente devido aos avanços recentes em métodos de armazenamento e processamento de volumes de dados em tempo quase real, típicos dos cenários do chamado Big Data e da popularização da Internet das Coisas (IoT).

Zude (2008) exemplifica o uso de técnicas ópticas no monitoramento de safras com o uso de comprimentos de onda fora do espectro visível - como o infravermelho próximo (NIR) - e a partir de técnicas de fluorescência e dispersão, por exemplo. Na indústria, diferentes técnicas vêm sendo aplicadas em diferentes cenários, como o controle da umidade - estimada através de índices de refração ou via espectros em comprimentos de onda específicos, como apresentado por Daikos and Scherzer (2021).

A utilização de técnicas de visão computacional juntamente com métodos de inteligência artificial tem encontrado vasto campo de aplicação, e a fotogrametria, como exposto anteriormente, também vem recebendo grande atenção acadêmica, especialmente nos últimos anos.

Entre os algoritmos de visão computacional mais largamente aplicados, o Detector de Bordas apresentado por Canny (1986), - e suas modificações - figura entre os mais importantes.

Através de um algoritmo de múltiplos estágios que utiliza o cálculo de variações como forma de otimizar a resposta da função, Canny demonstrou a possibilidade da detecção de bordas com boa resposta, boa localização e com o mínimo de ruídos.

Seu processamento inclui não apenas a utilização do Filtro Gaussiano, como o cálculo das magnitudes das intensidades após a determinação dos respectivos gradientes nas direções $\Delta x$ e $\Delta y$, e se utiliza etapas de supressão dos valores não-máximos em diferentes direções a partir de um pixel âncora, para diminuição dos ruídos.

A etapa final, de identificação de contornos, é conseguida a partir de análises de histerese na imagem - um processo bastante complexo, porém, capaz de apresentar resultados extraordinários, identificando contornos contínuos e descontínuos (Canny, 1986).

Os resultados encontrados para a aplicação do Filtro Binomial na imagem foram especialmente importantes, uma vez que foi conseguida uma diminuição de variância com magnitude mais de 13 vezes superior à da bibliotecapadrão, utilizada como comparação em imagem selecionada aleatoriamente.

Cruz (2015) havia notado o fato de que, se por um lado a CNAPap não fornece valores aritmeticamente exatos, eles apresentam um erro-padrão da média que se encontra em uma distribuição normal para um índice de confiança de $99 \%$ e que este valor converge quão maior seja o número de iterações - e, por isto sua opção pelo uso de 6 CNAPaps ligadas em série, com 20 iterações cada.

Não se deve menosprezar o fato de os valores de entrada considerados por si serem valores medidos, e que carregam consigo certo grau de incerteza - que seria ignorado caso os valores tivessem sido apenas somados, como ocorreria em uma convolução natural. Neste caso, o uso da LP que considera a contradição como componente importante - mostrou-se bastante eficaz.

Conquanto que o resultado tenha sido positivo, devese atentar que a necessidade do uso de CNAPaps em série e repetidas iterações traz como contraponto um tempo de processamento bastante elevado em comparação com o que teria sido conseguido através de uma convolução ordinária - fato este que sem dúvida deve ser considerado quando da opção pelo uso do modelo apresentado aqui.

Na etapa seguinte, ao contrário do que ocorreu com a suavização da imagem, a identificação das bordas da imagem trouxe mais ruídos do que o esperado, que precisaram ser corrigidos via código em momento posterior. Não há como fazer comparações diretas entre o método executado e o que teria sido conseguido via biblioteca, uma vez que ambos os princípios de funcionamento são bastante diferentes.

Inesperadamente, a combinação da utilização do ME$\mathrm{ENi}+$ MoBi na imagem previamente processada permitiu a identificação de defeitos nos objetos, o que não foi explorado aqui, mas que certamente poderia ser aperfeiçoado de forma a gerar mais um alarme de controle de qualidade, extremamente útil em uma linha de produção.

A etapa de extração das propriedades também mostrou ser positiva, com um erro encontrado para as duas figuras entre $0,1 \%$ e $1,77 \%$, ambos valores muito baixos em comparação com os valores nominais medidos pela régua de alumínio, o que demonstra um grande poder de aplicabilidade da técnica aqui desenvolvida em situações reais, práticas.

A classificação dos objetos via CNAPp também trouxe resultados satisfatórios e demandou apenas a execução de um algoritmo de baixa complexidade computacional, com limiares de resultados com boa separação - não havendo, entre os conjuntos de treinamento e teste - qualquer sobreposição entre eles. Sua taxa de acerto foi de $100 \%$ com o conjunto de teste, e seu $f$-score também representou o 
valor máximo, indicativo muito importante de seu sucesso.

Normalmente a classificação de imagens é realizada através de RNAs com algoritmos de backpropagation; estas, envolvem a definição de funções de ativação e funções de perda a partir das quais os mínimos locais são encontrados através de métodos numéricos de otimização através de redes multicamadas - isto é, com um número variável de neurônios, superior a 1.

A ideia do backpropagation envolve um processo de pelo menos duas fases: o cálculo dos erros, propagados entre as camadas de neurônios, e seu retorno para ajuste dos pesos sinápticos utilizados. Este processo é repetido pelo número de vezes necessário até que a classificação da imagem possa ser feita; isto, em contrapartida da execução única do algoritmo na CNAPp.

Além do backpropagation, imagens também são processadas através de redes neurais convolucionais (CNN) - que baseiam seu funcionamento em convoluções, tal como a abordagem deste projeto, e na estratégia de propagação de erros já mencionada - agora, no âmbito da aprendizagem profunda, que exige métodos computacionalmente mais intensivos.

Por fim, a identificação de desvios através da CNAPdi também trouxe como grande benefício a possibilidade de utilização de um algoritmo pouco intensivo, composto por apenas 8 passos.

A utilização do parâmetro área como alarme nos desvios se deu devido à sua maior sensibilidade à presença de erros nos lados das figuras do que o perímetro, uma vez que sua propagação é multiplicada. No cenário proposto, um objeto modificado manualmente com uma diferença de apenas $1 \mathrm{~mm}$ do objeto original - esperado - foi capaz de ativar um alarme de desvio, valor que representa menos de $1 \%$ do lado do objeto utilizado - importante indicativo de sua sensibilidade.

\section{Conclusões}

A aplicação de técnicas ópticas de monitoramento traz como principal benefício a possibilidade de execução em tempo próximo ao real e à distância, de forma automatizada, sem a necessidade de intervenção humana - como, por exemplo, a retirada de amostras para serem levadas a um laboratório e terem seus resultados utilizados como representativos de um lote. Aqui, o controle pode ser individualizado, com a geração contínua de relatórios e dados de medição sobre cada um dos objetos analisados.

A grande vantagem do uso da LP refere-se à consideração da incerteza como informação de importância na realização dos cálculos; uma vez que esta é inerente aos processos de medição e que seus erros são propagados, seus efeitos devem ser considerados durante a computação. Aqui, foi demonstrada sua plausibilidade, com resultados positivos.

Ao passo que este trabalho ajudou a preencher uma lacuna existente na literatura acadêmica sobre a utilização conjunta da LP com a fotogrametria, ele também permitiu identificar a geração de novas hipóteses de estudo e oportunidades de melhoria, abrindo a possibilidade para sua utilização em trabalhos futuros. Entre elas, mencionamse: i. Otimização dos custos computacionais do processamento de imagens utilizando a abordagem apresentada na UPC;

ii. Utilização do cálculo diferencial paraconsistente como forma de obtenção das derivadas primeiras entre os pixels durante a etapa de determinação das arestas, visto os algoritmos da LP não permitirem a utilização de valores negativos como graus de evidência, o que impediu, por exemplo, a aplicação direta de operadores como o Sobel e Scharr;

iii. Estudos sobre a aplicação do processamento paraconsistente de imagens para identificação de defeitos em objetos, efeito inesperado, porém observado durante a elaboração deste projeto;

iv. Realização de estudos com um número maior de amostras, como forma de validação da manutenção da efetividade em cenários mais complexos e sob condições com menor controle sobre os parâmetros;

v. Utilização de objetos com diferentes formas geométricas, mais complexas, para avaliação da manutenção das taxas de efetividade e determinação de diferentes limiares de classificação. Aqui, citam-se também os objetos com altura não-desprezível, em que os efeitos de profundidade podem influir nos resultados encontrados.

\section{Referências}

Albertz, J. (2001). Albrecht meydenbauer - pioneer of photogrammetric documentation of the cultural heritage, Proceedings 18th International Symposium CIPA, Alemanha.

Boroujeni, M., Sanimi, Y. and Roghaniam, E. (2021). Monitoring fuzzy linear quality profiles: A comparative study, International Journal of Industrial Engineering Computations 12: 37-48. http://doi.org/10.5267/j.ijiec. 2020. 10.002 .

Canny, J. (1986). A computational approach to edge detection, IEEE Transactions on Pattern Analysis and Machine Intelligence PAMI-8, 6: 679-698. http://doi.org/10. 1109/TPAMI . 1986.4767851.

Catalucci, S., Senin, N., Sims-Waterhouse, D., Ziegelmeier, S., Piano, S. and Leach, R. (2020). Measurement of complex freeform additively manufactured parts by structured light and photogrammetry, Measurement 164(108081). http://doi.org/10.1016/j .measurement . 2020.108081.

Cruz, C. M. d. (2015). Aplicação da rede neural artificial paraconsistente em controle estatístico de processos, Mestrado em Engenharia Mecânica, Faculdade de Engenharia Mecânica - Universidade Santa Cecília.

Da Silva Filho, J. I., Abe, J. and Torres, G, L. (2008). Inteligência Artificial com as Redes de Análises Paraconsistentes Teoria e Aplicações, LTC - Livros Técnicos e Científicos.

Da Silva Filho, J. I e Abe, J. (2001). Fundamentos das redes neurais artificiais paraconsistentes - Destacando Aplicações em Neurocomputação, Editora Arte e Ciência. 
Daikos, O. and Scherzer, T. (2021). Monitoring of the residual moisture content in finished textiles during converting by nir hyperspectral imaging, Talanta 221(121561). http://doi.org/10.1016/j.talanta.2020.121567.

El Masri, Y. and Rakha, T. (2020). A scoping review of nondestructive testing (ndt) techniques in building performance diagnostic inspections, Construction and Building Materials 265(120542). http: //doi .org/10.1016/j . conbuildmat.2020.120542.

Gonçalves, E. (2019). Desenvolvimento de um modelo estruturado em lógica paraconsistente para análise e tomada de decisão na escolha de materiais a serem montados nos protótipos e pré-produção de uma empresa montadora de veículos automotores, Mestrado em Engenharia Mecânica, Faculdade de Engenharia Mecânica - Universidade Santa Cecília.

Guidi, G., Malik, U. and Micoli, L. (2020). Optimal lateral displacement in automatic close-range photogrammetry, Sensors 20(6280). http://doi.org/10.3390/ s20216280.

Lopes, H. (2009). Aplicação de redes neurais artificiais paraconsistentes como método de auxílio no diagnóstico da doença de alzheimer, Mestrado em Ciências, Faculdade de Medicina - Universidade de São Paulo.

Ma, Y., Chen, G., Lv, M., Yi, W., Liu, X., Chen, H. and Zhu, B. (2021). Efficient and effective dimension control in automotive applications, IEEE Transactions on Industrial Informatics 17: 1583-1591. http://doi.org/10.1109/TII. 2020. 2990168.

Martinelli, F. B. (2009). Gestão da qualidade total, IESDE Brasil.

Menna, F., Nocerino, E., Remondino, F., Saladino, L. and Berri, L. (2020). Towards online uas-based photogrammetric measurements for $3 \mathrm{~d}$ metrology inspection, Photogrammetric Record . http://doi.org/10.1111/phor. 12338.

Mário, M., Da Silva Filho, J., Damin, O. and Lopes, R. (2012). Modelo digital com interação do usuário para obtenção de medidas de árvores utilizando lógica paraconsistente anotada, Revista Seleção Documental 28.

Narvydas, E. and Puodziuniene, N. (2020). Curved beam stress analysis using a binary image of the cross-section, Computer Applications in Engineering Education 28: 16961707. http://doi.org/10.1002/cae.22348.

Odumosu, J., Adeleke, O., Nnam, V., Avoseh, O. and Ogundeji, O. (2020). Stereophotogrammetry for 2-d building deformation monitoring using kalman filter, Reports on Geodesy and Geoinformatics 110: 1-7. http://doi .org/ 10.2478/rgg-2020-0006.

Paladini, E. P. (2011). Avaliação Estratégica da Qualidade, 2 edn, Atlas.

Rocha, D. A. P. d. (2019). Aplicação de métodos estatísticos com apoio da lógica paraconsistente para o estudo de caso da confiabilidade de ativos industriais, Mestrado em Engenharia Mecânica, Faculdade de Engenharia Mecânica - Universidade Santa Cecília.
Souza, S. (2013). Sistema de reconhecimento de caracteres numéricos manuscritos baseado nas redes neurais artificiais paraconsistentes, Mestrado em medicina, Faculdade de Medicina da Universidade de São Paulo.

Stachniss, C. (2020). Photogrammetry i \& ii, Notas de aula, University of Bonn, Alemanha.

Tan, C., Feng, S., Zhao, X., Shan, X. and SL, F. (2021). Longitudinal variations in channel morphology of an ephemeral stream from upland to lowland, daihai lake basin, north china, Geomorphology 372(107450). http: //doi.org/10.1016/j.geomorph.2020.107450.

Tarolli, P., Pijil, A., Cucchiaro, S. and Wei, W. (2020). Slope instabilities in steep cultivation systems: Process classification and opportunities from remote sensing, Land Degradation \& Development . http://doi .org/10.1002/ ldr.3798.

Wang, J., Tao, B., Gong, Z., Yu, S. and Yin, Z. (2021). A mobile robotic measurement system for large-scale complex components based on optical scanning and visual tracking, Robotics and Computer-Integrated Manufacturing 67(102010). http://doi.org/10.1016/j.rcim. 2020. 102010.

Wu, B., Li, Y., Liu, W., Want, Y., Li, F., Zhao, Y. and H, Z. (2021). Centimeter-resolution topographic modeling and fine-scale analysis of craters and rocks at the chang'e-4 landing site, Earth and Planetary Science Letters 553(116666). http://doi.org/10.1016/j.epsl. 2020.116666.

Zollini, S., Alicandro, M. and Giallonardo, M. (2020). Uav photogrammetry for concrete bridge inspection using object-based image analysis (obia), Remote Sensing 12(3180). http://doi.org/10.3390/rs12193180.

Zude, M. (2008). Optical Monitoring of Fresh and Processed Agricultural Crops, CRC Press. 